\title{
Poster Abstract: Low-Power Wireless Channel Quality Estimation in the Presence of RF Smog
}

\author{
Anwar Hithnawi, Hossein Shafagh \\ Department of Computer Science \\ ETH Zurich \\ \{hithnawi, shafagh\}@inf.ethz.ch
}

\author{
Simon Duquennoy \\ SICS Swedish ICT AB \\ Kista, Sweden \\ simonduq@sics.se
}

\begin{abstract}
Low-power wireless networks deployed in indoor environments inevitably encounter high-power Cross Technology Interference (CTI) from a wide range of wireless devices operating in the shared RF spectrum bands. This severely reduces the performance of such networks and possibly causes loss of connectivity, which affects their availability and drains their resources. In this work, to address the channel uncertainty, a consequence of CTI, we propose a novel channel metric that $(i)$ harnesses the local knowledge of a node about the wireless channel to discern the presence of persistent high-power interferers, and (ii) assists the node in inferring its proximity to the dominant interference sources in the physical space. In order to motivate and validate the necessity of such a metric, we empirically characterize the impact of the interaction between high/lowpower cross technology interferers and IEEE 802.15.4.
\end{abstract}

\section{INTRODUCTION}

An increased amount of low-power wireless devices are envisioned to be deployed in densely populated areas in the near future to form the communication backbone of the Internet of Things (IoT). These devices are expected to endure interference from other RF technologies operating in the lightly regulated, yet crowded, ISM bands. Cross Technology Interference (CTI) introduces high uncertainty and complexity to the wireless channel, which particularly affects the lowpower wireless networks given their scarce resources and low transmission power.

In this paper, we focus on high-power CTI interferers such as analog phones, digital cordless phones, wireless cameras, and microwave ovens. Unlike CTI from protocol technologies such as IEEE 802.11 and Bluetooth, the impact and mitigation approaches of high-power interferers were not adequately addressed for low-power wireless communication. Moreover, these technologies were designed to meet a certain level of coexistence within technologies based on the same PHY. However, less work has focused on harmonizing coexistence across technologies. High-power interferers are persistent, broadband and emit at a power that is several orders of magnitude higher than those of 802.15.4. Hence, they are less amenable to coexistence with low-power networks. Conventional countermeasures to mitigate CTI take two directions: (i) Avert interferer frequencies, by active spectrum sampling to identify interference free channels or classifying the interference source for a better selection of channels that do not overlap with the interferer's channels. However the spectrum is increasingly dense with technologies that access the medium with wider bands and have a time-variant nature, which would

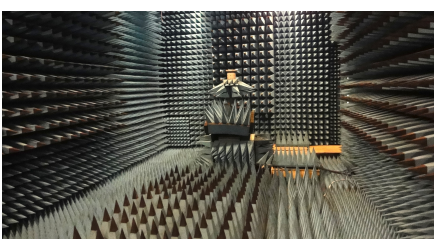

(a) Anechoic room

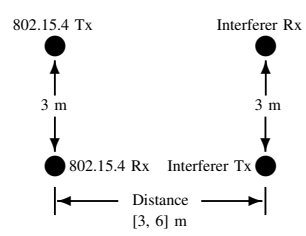

(b) Experiment setup
Fig. 1. Experiment setup for the CTI impact study in an anechoic room.

make it challenging to find interference free channels. (ii) Use of resilience coding schemes or partial packet recovery mechanisms [1], [2]. These mechanisms are ineffective in the presence of high-power CTI, as the problem stems from complete loss of connectivity. Recent research has introduced mechanisms for interference cancellation [3]. Such approaches are desirable as they facilitate spectral usage efficiency and yet harmonize coexistence across different technologies. However, such solutions are not yet feasible for low-power networks due to their computational requirements.

In this ongoing work, we empirically characterize the impact of high and low-power CTI on 802.15.4 networks. Driven by this CTI study, we propose a channel metric that harnesses the node's local knowledge of the wireless channel to discern the presence of persistent high-power interferers. We discuss how such an indicator can be exploited by interferenceaware protocols. Moreover, we show how nodes can infer their proximity to the dominating interference sources with respect to their neighboring nodes, and hence, potentially allowing affected nodes to route away from the interference source.

\section{IMPACT OF CROSS-TECHNOLOGY INTERFERENCE ON 802.15 .4}

We run a first set of experiments to quantify the impact of CTI on the performance of 802.15.4 networks. We focus our experiments on a set of interfering technologies operating in the $2.4 \mathrm{GHz}$ ISM band which are pervasive in today's environment. In order to have full control on the source of errors and to isolate the impact of surrounding interference sources, we ran our experiments in an anechoic chamber with an experiment setup as illustrated in Figure 1.

Figure $2 \mathrm{a}$, shows the power profiles for a microwave oven, a camera, and an analog phone. We use USRP N210 to collect these power profiles. We have two TelosB nodes transmitting 100 byte frames at $100 \mathrm{~ms}$ interval with carrier sense enabled, 


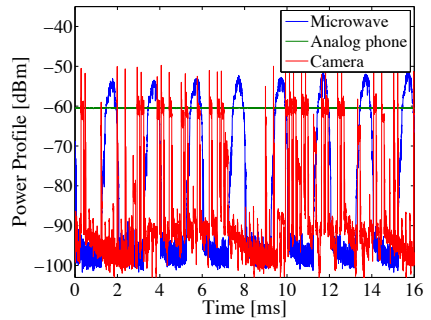

(a) Power Profiles

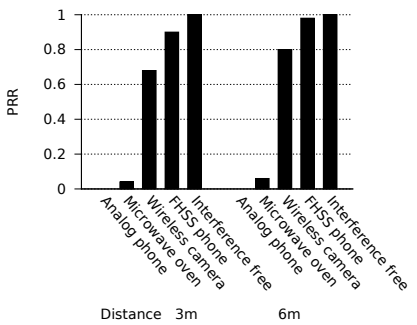

(b) PRR

Fig. 2. Characteristics of different interference sources measured in an anechoic room. (a) Power profiles for microwave oven, analog cordless phone, and digital wireless camera. (b) PRR of 802.15.4 links with presence of CTI in interleaved frequencies, at two different distances.

and vary the position of the receiver, either $3 \mathrm{~m}$ or $6 \mathrm{~m}$ away from the interference source. Figure $2 b$ shows the measured Packet Reception Rate (PRR). In the presence of high-power interferers, the 802.15.4 nodes experienced severe losses. For the analog phone, they were unable to communicate at all as they backed off constantly.

\section{PRELIMINARY DESIGN AND INITIAL EXPERIMENTS}

We propose a metric that relies on fine-grained measurements of the energy in the channel to detect the presence of high-power interferers, and classify them in terms of persistence and power. Nodes in the 802.15.4 network monitor the occupancy of the channel passively, at a high frequency. This monitoring can be triggered for example after observing a given number of successive transmission failures. In contrast with channel metrics such as ETX, this metric does not require exchanged packets to recognize the degradation in the link quality, which makes it more agile, particularly to time-variant interferers.

Characterizing High-Power Interferers. To detect the persistence of the interference source, we measure the portion of the $R S S_{i}$ samples in the time interval $T_{s}$ that exceeds the base noise level $N_{\mathrm{CCA}}$ ( $-45 \mathrm{dBm}$ in our experiments). For ratios higher than a threshold ( 0.9 in our experiments), the interferer is considered persistent. To discern high-power interferers, we compute the induced power level on the channel as the mean deficit between $R S S_{i}$ and $N_{C C A}$. For power levels higher than a fixed threshold, the interferer is considered high-power.

Locating High-Power Interferers. Upon detecting the presence of high-power interferers, the node employs signal strength observations to identify its proximity with respect to the interferer. Given that a node lacks the knowledge of the interferer's transmission power, it infers its proximity to the interferer based on the difference of power level induced on neighboring nodes. To this end, nodes exchange information on the induced power level with neighboring nodes: the vector $R S S=\left[r \hat{s} s_{1} \ldots r s \hat{s}_{N}\right]$, where $N$ is the number of neighboring nodes and $r \hat{s} s_{i}$ is the mean of the induced power level on the neighbor $i$. The successful delivery of at least a subset of this vector is required. Based on this information, the node learns the impact of the interferer on other nodes and can locally estimate its relative proximity to the interferer. A node could potentially exploit this information to locally select a route that steers away from the interferer.

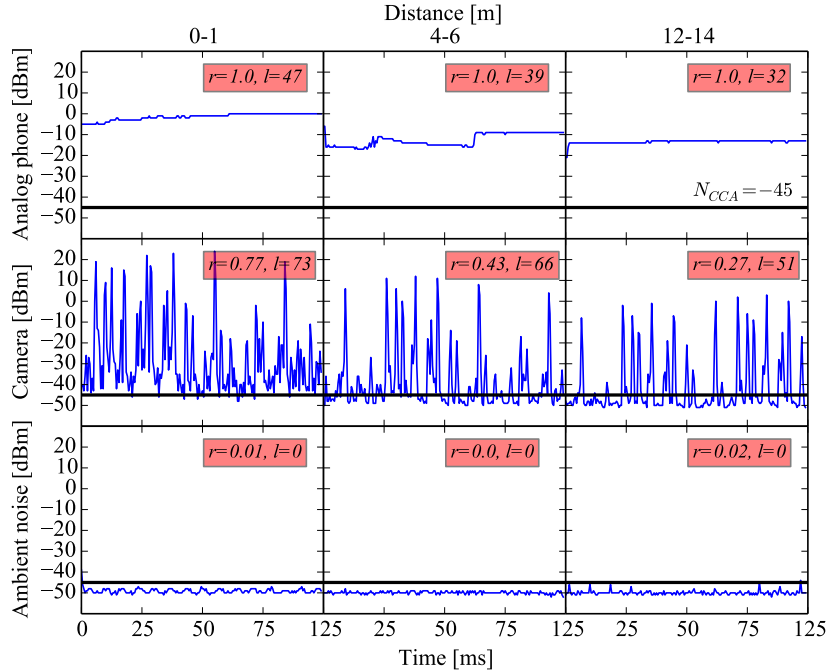

Fig. 3. Noise sampled by a TelosB node at $62.5 \mathrm{kHz}$ for different interference sources, and resulting link quality estimates $(r$ : persistence ratio, $l$ : power level). The distance from the interference source not only affects the induced power level but also, in the case of camera, the persistence of interference.

Initial Experiments. We perform a set of experiments in the basement of our building (least WiFi-crowded area in the building). We instrumented a TelosB mote to passively monitor the radio medium by logging fine-grained readings of noise, sampled at $62.5 \mathrm{kHz}$ (the symbol frequency). We run the experiments with different sources: an analog phone, a wireless camera, and without interference. We position the TelosB node at distances ranging from 0 to $14 \mathrm{~m}$ to the interference source. Figure 3 summarizes our experiments, and for each setting shows the persistence and power level as measured by the node. Our results show that the sampling frequency of $62.5 \mathrm{kHz}$ suffices to correctly classify the analog phone as persistent, and the camera as non-persistent. Also interesting is the observation that the nearly constant power output of the analog phone results in a perceived persistence that is independent from the distance to the source. This is in contrast with the camera's more bursty output, which results in a persistence that decreases gradually as distance increases. We argue that protocols can benefit from this two-dimensional information (persistence, power level) to better react to CTI.

\section{FUTURE WORK}

Motivated by initial results, we outline our future work by: (i) evaluating the accuracy of our metric of node-based channel information, (ii) evaluating the impact of using our node local metric for channel assessment in harmless channel occupancy, back-off strategies, and for the purpose of routing away from interference sources.

\section{REFERENCES}

[1] C. Liang, N. Priyantha, J. Liu, A. Terzis, "Surviving Wi-Fi Interference in low Power ZigBee Networks," in ACM SenSys, 2010.

[2] A. Hithnawi, "Exploiting Physical Layer Information to Mitigate CrossTechnology Interference Effects on Low-Power Wireless Networks," in ACM Sensys, 2013.

[3] S. Gollakota, F. Adib, D. Katabi, and S. Seshan, "Clearing the RF Smog: Making 802.11n Robust to Cross-Technology Interference," in SIGCOMM, 2011. 